\title{
MULTI-CHANNEL TIME ENCODING FOR IMPROVED RECONSTRUCTION OF BANDLIMITED SIGNALS
}

\author{
Karen Adam, Adam Scholefield, Martin Vetterli
}

\author{
School of Computer and Communication Sciences \\ Ecole Polytechnique Fédérale de Lausanne (EPFL)
}

\begin{abstract}
Traditional sampling involves encoding a signal through (time, value)-pairs. In contrast, time encoding machines (TEMs) characterize a signal by recording time points which depend on the integral of the signal over time. We study multi-channel TEMs where channels have shifted values for their integrators. We show that $M$ channels can enable recovery of bandlimited signals with $M$ times the bandwidth of that allowed in the single channel case. Moreover, our recovery algorithm is linear, even when the shift between the integrators of the TEMs is unknown. This is in stark contrast to traditional multi-channel sampling, where complicated non-linear methods are required to recover the unknown time shift between channels.
\end{abstract}

Index Terms - Bandlimited signals, sampling methods, signal reconstruction.

\section{INTRODUCTION}

Biology and engineers have arrived at two very different ways to sample continuous signals. Whilst almost all human-made systems are based on the idea of sampling a signal's amplitude at certain time points, in biology, a signal can be encoded using the timings of action potentials generated by neurons. Therefore, it is natural to wonder if we can design improved sampling systems by taking inspiration from nature.

Traditional (time, value)-pair sampling is dominated by uniform sampling of bandlimited signals [1]. More recently, sampling results have been developed for other signal classes such as general shift-invariant subspaces [2], unions of subspaces [3], and signals of finite rate of innovation [4]. The extension beyond uniform sampling includes results for irregular samples [5], jittered samples [6], samples at unknown locations [7, 8] and incomplete frequency samples [9]. Whilst these results are of both theoretical importance and practical interest, they diverge from the example set by biology.

Time encoding is a more neuromorphic approach to sampling. In a nutshell, a neuron samples its input by integrating it (spatially and temporally) and emitting action potentials when the integral reaches a threshold [10]. A time encoding machine (TEM) also integrates the incoming signal and compares the integral with a threshold [11]. Each time a threshold is reached, time points are recorded, and these recordings are comparable to action potentials emitted by neurons. TEMs can be made even more biologically plausible by considering leaky integrate-and-fire neuron models with refractory period [12] or even Hodgkin-Huxley neurons [13]; however, in this paper, we consider a basic definition including a perfect integrator with a reset operation.

In the traditional sampling setup, Papoulis extended Shannon's theory to the multi-channel case by showing that, if a bandlimited signal is input to $M$ linear shift-invariant systems, perfect reconstruction is possible with $1 / M$ times the sampling rate per channel [14]. Likewise, it is interesting to investigate how multiple TEMs (or neurons) can improve signal representation. In biology, it seems intuitive that multiple neurons encode a signal better than one.

In this paper, we study the reconstruction performance of multi-channel TEMs for bandlimited signals. In the single channel case, reconstruction of bandlimited signals from time encoding was studied in [11]. In a similar spirit to Papoulis, Lazar et. al. later considered multi-channel TEMs, where each channel is prefiltered with a linearly independent filter before time encoding $[12,15]$. To reconstruct, each channel is independently decoded reproducing that channel's postfiltered continuous-time signal and, finally, these are combined to reproduce the original signal.

In contrast, we consider multi-channel TEMs with an unknown shift in their integrators. This is particularly relevant in hardware and biology as it is normally not possible to perfectly align the channels. A similar problem occurs in traditional multi-channel sampling, where it is normally impossible to ensure each channel samples at the desired time-points. To date, this has only been solved in special cases with complex non-linear algorithms [16]. We show that, in the TEM case, a linear reconstruction algorithm can recover bandlimited signals sampled using multi-channel time encoding, even if the inter-integrator shifts are unknown.

In the rest of this paper, we recap the basics of TEMs and introduce the multi-channel setup. Then, we present a reconstruction algorithm for two-channel time encoding and finally generalize the results to the $M$-channel case. 


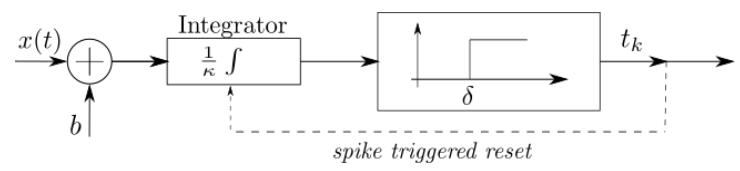

Fig. 1. TEM circuit. The input is biased and integrated until the integral reaches a threshold. Once the threshold is reached, a spike is created and the integrator value is reset ${ }^{2}$.

\section{SINGLE-CHANNEL TIME ENCODING}

Whilst this section summarizes results from [11], the TEM setup we use differs slightly from the one put forward in [11]. In fact, if we assume no refractory period, our setup is equivalent to the encoding scheme presented in [17]. As depicted in Fig. 1, the proposed TEM has three parameters $\kappa, \delta$, and $b$. It takes as input a bounded signal $x(t)$, such that $|x(t)| \leq c<b$, and outputs a spike train as follows: the machine adds $b$ to its input, scales the sum by $1 / \kappa$ and passes the result through an integrator. Once the integrator output reaches the threshold $\delta$, a spike is recorded and the value of the integrator is reset to $-\delta$. In other words, if a spike was recorded at $t_{k}$, then the next spike time $t_{k+1}$ will satisfy

$$
\frac{1}{\kappa} \int_{t_{k}}^{t_{k+1}}(x(u)+b) d u=2 \delta .
$$

The spikes times $t_{k}, k \in \mathbb{Z}$ will be used for signal reconstruction. An example of a TEM sampling is depicted in Fig. 2.

Assume that the input signal is a $2 \Omega$-bandlimited signal; i.e., its Fourier transform is zero for $|\omega| \in(\Omega, \infty)$. In [11], Lazar and Tóth show that perfect reconstruction is possible if

$$
\Omega<\frac{\pi(b-c)}{2 \kappa \delta} .
$$

To see why this is true, first note that the following integrals can be computed from the trigger times:

$$
\int_{t_{k}}^{t_{k+1}} x(u) d u=2 \kappa \delta-b\left(t_{k+1}-t_{k}\right),
$$

where $t_{k}$ and $t_{k+1}$ are any two consecutive trigger times. Next, define the following reconstruction operator $\mathcal{A}$ :

$$
\mathcal{A} x(t)=\sum_{k \in \mathbb{Z}} \int_{t_{k}}^{t_{k+1}} x(u) d u g\left(t-s_{k}\right),
$$

where $s_{k}=\left(t_{k}+t_{k+1}\right) / 2$ and $g(t)=\sin (\Omega t) /(\pi t)$.

One can then iteratively estimate $x(t)$ as follows:

$$
x_{0}=\mathcal{A} x, \quad x_{l+1}=x_{l}+\mathcal{A}\left(x-x_{l}\right) .
$$

By induction, one can show that the $l^{\text {th }}$ estimate is a partial sum of a Neumann series: $x_{l}=\mathcal{A} \sum_{k=0}^{l}(\mathcal{I}-\mathcal{A})^{k} x$, which converges to $\lim _{l \rightarrow \infty} x_{l}=\mathcal{A A}^{-1} x=x$, if

$$
\|\mathcal{I}-\mathcal{A}\|<1 .
$$

\footnotetext{
${ }^{2} \mathrm{~A}$ similar figure appeared in [17].
}

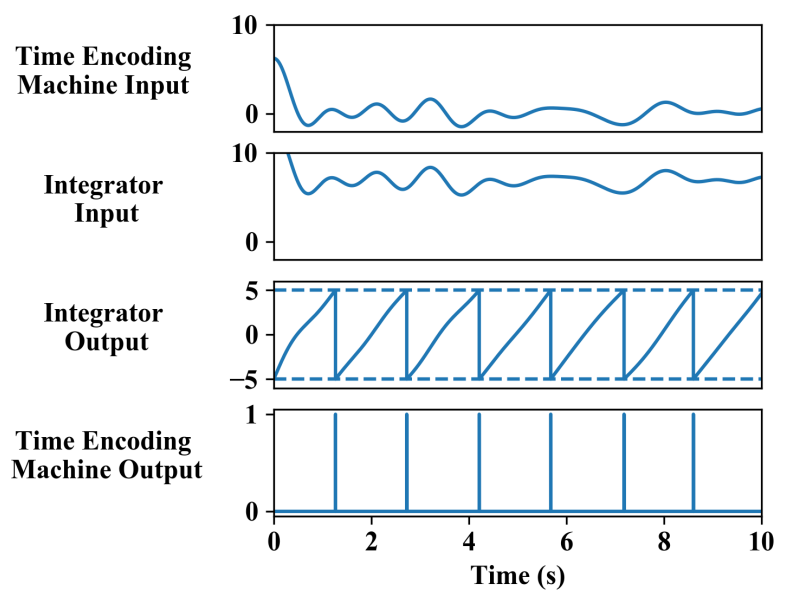

Fig. 2. TEM processing of signal. From top to bottom, the input signal, the biased signal input to the integrator, the output of the integrator and the final output. The dashed lines in the third figure mark the threshold $\delta$ and the reset level $-\delta$.

Here, $\|$.$\| denotes the operator norm.$

The following lemma provides a bound for $\|\mathcal{I}-\mathcal{A}\|$.

Lemma 2.1 (Lazar and Tóth 2004).

$$
\|\mathcal{I}-\mathcal{A}\| \leq \frac{\Omega}{\pi}\left(\sup \left(t_{k+1}-t_{k}\right)\right) .
$$

Proof. The proof requires Bernstein's and Wirtinger's inequalities: see proofs of Lemmas 2 and 3 in [11].

To bound the separation between spike times, we recall that $|x(t)| \leq c$, which, when substituted into (2), yields

$$
\begin{aligned}
-c\left(t_{k+1}-t_{k}\right) & \leq 2 \kappa \delta-b\left(t_{k+1}-t_{k}\right), \\
t_{k+1}-t_{k} & \leq \frac{2 \kappa \delta}{b-c} .
\end{aligned}
$$

Together, these results lead to the following theorem.

Theorem 2.1 (Lazar and Tóth 2004). Assume $x(t)$ is a $2 \Omega$ bandlimited signal that is bounded such that $|x(t)| \leq c$. If $x(t)$ is passed through a TEM that has parameters such that $b>c$ and $\Omega$ satisfies (1), then

$$
\lim _{l \rightarrow \infty} x_{l}(t)=x(t) .
$$

Proof. Combine Lemma 2.1 with (7) and the condition for the convergence of Neumann series in (5).

Therefore, in the case of a bandlimited signal with bandwidth $2 \Omega$, an iterative reconstruction algorithm can guarantee convergence to the original signal, given that the parameters of the TEM satisfy (1).

A closed form solution for the problem can also be devised. First, let $\mathcal{G}$ be the operator defined as

$$
\mathcal{G} \mathbf{y}=\sum_{k \in \mathbb{Z}} y_{k} g\left(t-s_{k}\right) \text {. }
$$


In addition, define $\mathbf{q}=\left[\int_{t_{k}}^{t_{k+1}} x(u) d u\right]_{k \in \mathbb{Z}}$, and $\mathbf{H}=$ $\left[H_{l k}\right]_{l, k \in \mathbb{Z}}=\left[\int_{t_{l}}^{t_{l+1}} g\left(u-s_{k}\right) d u\right]_{l, k \in \mathbb{Z}}$. Then, one can write $x(t)=\mathcal{G} \mathbf{H}^{+} \mathbf{q}$ where $\mathbf{H}^{+}$is the pseudoinverse of $\mathbf{H}$. We refer the reader to [11] for a proof.

\section{MULTI-CHANNEL TIME ENCODING}

Consider two TEMs A and $\mathrm{B}$, with the same parameters $\kappa$, $\delta$, and $b$. At first sight, it seems that A and B will output the same encoding of an input signal $x(t)$. However, consider a scenario where the integrator of $\mathrm{A}$ is always $\alpha \neq 0$ ahead of the integrator of B (modulo $2 \delta$ ). Then, the threshold is reached at different times in the two machines. Therefore, the recorded spike times of $x(t)$ are different and the overall encoding of the signal is different.

Intuition tells us that we can probably gain more information about $x(t)$ by considering the outputs of both machines.

More generally, our multi-channel time encoding setup assumes that a bandlimited signal is passed through $M$ TEMs which are shifted with respect to each other with shifts $\alpha_{i}$.

We will show that reconstruction of a $2 \Omega$-bandlimited signal is possible if

$$
\Omega<\frac{\pi(b-c)}{\kappa \bar{\alpha}},
$$

where $\bar{\alpha}$ is the maximum shift between any two adjacently spiking machines. For such cases, we will provide a reconstruction algorithm which does not need to know the $\alpha_{i}$ 's.

Using $M$ TEMs to encode a bandlimited signal, one can reconstruct a signal with bandwidth which is $M$ times larger than in the single channel case, when $\alpha_{i}=\bar{\alpha}=2 \delta / M, \forall i=$ $1 \cdots M$. In future work, we hope to show that the bandwidth can always be reduced by $M$ no matter the values of the shifts (as long as they are nonzero).

Practically, this result indicates that multi-channel sampling and reconstruction using TEMs does not require the precise setting, or even knowledge, of inter-integrator shifts. Moreover, clock synchronization, which poses an issue in multi-channel amplitude sampling, can be entirely bypassed here because the outputs of all the TEMs can be summed (in hardware) into one final spike train. This spike train is fed as input to the decoding machine (see Fig. 3). We will derive a reconstruction algorithm assuming two channels with unknown shift by first assuming the shift is known and then rearranging our reconstruction operator. Then, we extend this to the $M$-channel case with unknown shifts.

\subsection{Two-channel time encoding and decoding}

Assume we have two shifted machines A and B encoding the same signal and suppose, without loss of generality, that B's integrator is $\alpha_{1}$ ahead of A's (modulo $2 \delta$ ). It follows that A's integrator is $\alpha_{2}=2 \delta-\alpha_{1}$ ahead of B's (modulo $2 \delta$ ), and that the spikes of A and B are interleaved in time: there will

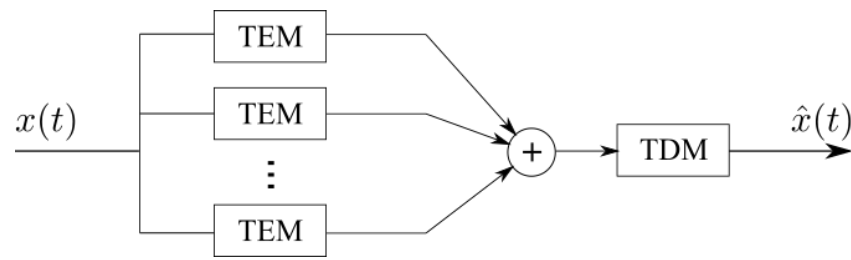

Fig. 3. Multi-channel time encoding and decoding. The signal goes through different TEMs, the outputs of which are combined through an adder and fed into one time decoding machine (TDM) which reconstructs the signal.

always be one spike from B between any two spikes of A, and vice versa. Let the indices of the spike times be such that $t_{k}^{(\mathrm{A})}<t_{k}^{(\mathrm{B})}, \forall k \in \mathbb{Z}$, where $t_{k}^{(\mathrm{A})}$ and $t_{k}^{(\mathrm{B})}$ denote, respectively, the spike times emitted by machines A and $\mathrm{B}$.

The integral of the signal between any pair of consecutive spike times will satisfy

$$
\begin{gathered}
\int_{t_{k}^{(\mathrm{A})}}^{t_{k}^{(\mathrm{B})}} x(u) d u=\kappa \alpha_{1}-b\left(t_{k}^{(\mathrm{B})}-t_{k}^{(\mathrm{A})}\right), \\
\int_{t_{k}^{(\mathrm{B})}}^{t_{k+1}^{(\mathrm{A})}} x(u) d u=\kappa \alpha_{2}-b\left(t_{k+1}^{(\mathrm{A})}-t_{k}^{(\mathrm{B})}\right) .
\end{gathered}
$$

If we now combine and order the spike times $t_{k}^{(\mathrm{A})}$ and $t_{k}^{(\mathrm{B})}$ into one set of spike times $\left\{\tilde{t}_{k}: k \in \mathbb{Z}\right\}$, then we can bound the separation between any pair of spikes, just like we did in the single channel case. In particular, substituting $|x(t)| \leq c$ into (10) and (11) yields

$$
t_{k}^{(\mathrm{B})}-t_{k}^{(\mathrm{A})} \leq \frac{\kappa \alpha_{1}}{b-c} \quad \text { and } \quad t_{k+1}^{(\mathrm{A})}-t_{k}^{(\mathrm{B})} \leq \frac{\kappa \alpha_{2}}{b-c},
$$

respectively. Setting $\bar{\alpha}=\max \left(\alpha_{1}, \alpha_{2}\right)$ gives

$$
\tilde{t}_{k+1}-\tilde{t}_{k} \leq \frac{\kappa \bar{\alpha}}{b-c} .
$$

We can therefore define the same reconstruction operator as in (3), but taking into account the new combined set of spike times $\left\{\tilde{t}_{k}: k \in \mathbb{Z}\right\}$, with corresponding midpoints $\tilde{s}_{k}$ :

$$
\mathcal{A} x(t)=\sum_{k \in \mathbb{Z}} \int_{\tilde{t}_{k}}^{\tilde{t}_{k+1}} x(u) d u g\left(t-\tilde{s}_{k}\right) .
$$

Since the integrals in (13) are over the intervals $\left[\tilde{t}_{k}, \tilde{t}_{k+1}\right]$, computing them requires knowledge of the $\alpha_{i}$ 's. To remove this dependence, define $f_{l}(t)=\sum_{k<l}(-1)^{k-l} g\left(t-\tilde{s}_{k}\right), l \in$ $\mathbb{Z}$, so that $g\left(t-\tilde{s}_{k}\right)=g_{k}(t)=f_{k}(t)+f_{k+1}(t)$. Then,

$$
\begin{aligned}
\mathcal{A} x(t) & =\sum_{k \in \mathbb{Z}} \int_{\tilde{t}_{k}}^{\tilde{t}_{k+1}} x(u) d u \quad\left(f_{k}(t)+f_{k+1}(t)\right) \\
& =\sum_{k \in \mathbb{Z}} \int_{\tilde{t}_{k}}^{\tilde{t}_{k+2}} x(u) d u f_{k+1}(t) .
\end{aligned}
$$


Notice that, since $\int_{\tilde{t}_{k+2}}^{\tilde{t}_{k}} x(u) d u=2 \kappa \delta-b\left(\tilde{t}_{k+2}-\tilde{t}_{k}\right)$, the operator $\mathcal{A}$ can now be applied without knowing the shifts $\alpha_{i}$.

Lemma 2.1 still holds for $\mathcal{A}$, and, as before, the iterative algorithm converges if $\|\mathcal{I}-\mathcal{A}\|<1$. Therefore, one can reconstruct the bandlimited signal if (9) is satisfied.

As for the closed form solution, following the treatment in [11], we can write

$$
x(t)=\mathcal{G} \mathbf{B}^{-1}\left(\mathbf{B H B}^{-1}\right)^{+} \mathbf{B q},
$$

where $\mathcal{G}, \mathbf{H}$, and $\mathbf{q}$ are as defined before (changing $t_{k}$ to $\tilde{t}_{k}$ ), and $\mathbf{B}=\left[B_{k l}\right]_{k, l \in \mathbb{Z}}$ where $B_{k l}=1$ if $k=l$ or $k=l+1$ and zero otherwise. Note that, here, $\mathbf{B q}$ is computed as one vector, which does not require the knowledge of the $\alpha_{i}$ 's.

We have thus found both a recursive and a closed form solution for the estimation of a signal from spike times obtained from two TEMs with an unknown nonzero shift, and have shown that the bandwidth bound is now inversely proportional to the shift $\bar{\alpha}$ (where $\delta \leq \bar{\alpha}<2 \delta$ ). Thus, in the ideal machine spacing, two channels can perfectly reconstruct a signal that has double the bandwidth as that allowed in the single-channel case. This occurs when $\alpha_{1}=\alpha_{2}=\delta$.

\subsection{M-channel time encoding and decoding}

We can extend the above algorithm to the case of $M$-channel time encoding. Let $\mathrm{A}_{1}, \cdots, \mathrm{A}_{M}$ be TEMs encoding the same signal, ordered such that $t_{k}^{\left(\mathrm{A}_{i}\right)}<t_{k}^{\left(\mathrm{A}_{j}\right)}, \forall k \in \mathbb{Z}$ if $i<j$. They have the same parameters $\kappa, \delta$, and $b$, and the inter-integrator shifts $\alpha_{1}, \alpha_{2}, \cdots, \alpha_{M}$ correspond, respectively, to the shifts between $\mathrm{A}_{1}$ and $\mathrm{A}_{2}, \mathrm{~A}_{2}$ and $\mathrm{A}_{3}, \cdots$, and $\mathrm{A}_{M}$ and $\mathrm{A}_{1}$.

Let $\left\{\tilde{t}_{k}: k \in \mathbb{Z}\right\}$ be the combined and ordered spike times from all machines, and $\bar{\alpha}=\max _{1 \leq i \leq M} \alpha_{i}$. Now let $\mathcal{A}$ be defined as in (13). As in Section 3.1, $\mathcal{A}$ can be rewritten as

$$
\mathcal{A} x(t)=\sum_{k \in \mathbb{Z}} \int_{\tilde{t}_{k}}^{\tilde{t}_{k+M}} x(u) d u f_{k+M-1}(t),
$$

where $f_{l}(t)$ is such that $g\left(t-\tilde{s}_{k}\right)=\sum_{l=k}^{k+M-1} f_{l}(t)$. Now define $\mathbf{B}=\left[B_{k l}\right]_{k, l \in \mathbb{Z}}$ and $B_{k l}=1$ for $k=l+i$ where $i=$ $0, \cdots, M-1$, and 0 otherwise. The closed form estimation for the original signal is then done as in (15).

Let $x_{l}(t)$ be the $l^{t h}$ estimate of $x(t)$ obtained using the recursive algorithm from (4), based on the operator $\mathcal{A}$ in (16), then we have the following theorem.

Theorem 3.1. Assume $x(t)$ is a $2 \Omega$-bandlimited signal that is bounded such that $|x(t)| \leq c$. If $x(t)$ is passed through an $M$-channel TEM that has parameters such that $b>c$ and $\Omega$ satisfies (9), then

$$
\lim _{l \rightarrow \infty} x_{l}(t)=x(t) .
$$

Proof. Our operator in (16) performs the same operation as the one defined in (3), substituting $t_{k}$ for $\tilde{t}_{k}$.

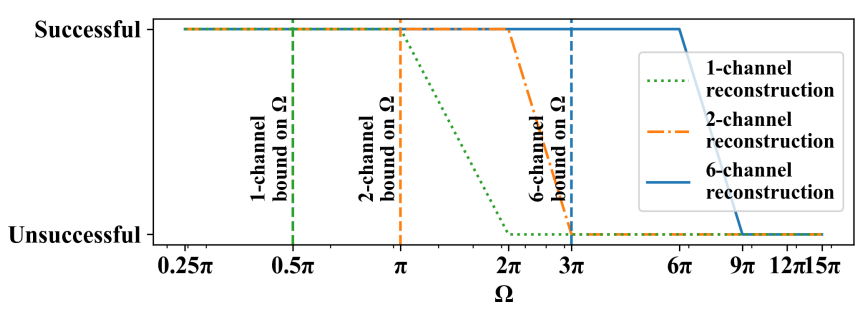

Fig. 4. Reconstruction success when $M=1,2$ and 6 channels with ideal inter-integrator shifts encode a signal as its bandwidth $2 \Omega$ varies. The $\Omega$ axis is plotted in $\log$ scale. Success is based on having a low normalized mean squared error when averaged over 2000 randomly generated $2 \Omega$-bandlimited signals.

We recall that our recursive algorithm using operator $\mathcal{A}$ converges if $\|\mathcal{I}-\mathcal{A}\|<1$ and that Lemma 2.1 places a bound on this norm. Therefore, we only require the right-hand side in (6) to be less than 1 . To show that this holds if (9) is true, we note that $\int_{t_{k}^{\left(\mathrm{A}_{i}\right)}}^{t^{\left(\mathrm{A}_{i+1}\right)}} x(u) d u=\kappa \alpha_{i}-$ $b\left(t_{k}^{\left(\mathrm{A}_{i+1}\right)}-t_{k}^{\left(\mathrm{A}_{i}\right)}\right)$, if $1 \leq i<M$, and $\int_{t_{k}^{\left(\mathrm{A}_{M}\right)}}^{t^{\left(\mathrm{A}_{1}\right)}} x(u) d u=$ $\kappa \alpha_{M}-b\left(t_{k+1}^{\left(\mathrm{A}_{1}\right)}-t_{k}^{\left(\mathrm{A}_{M}\right)}\right)$, so that we can bound $\tilde{t}_{k+1}-\tilde{t}_{k} \leq$ $\kappa \bar{\alpha} /(b-c)$.

We have thus shown that using $M$-channel time encoding can allow us to encode a signal with $M$ times the bandwidth as that allowed in the single channel case. This occurs when all channels have integrators which are equally spaced, i.e. $\bar{\alpha}=2 \delta / M=\alpha_{i}, \forall i=1, \cdots, M$.

We illustrate, in Fig. 4, the improvement of signal reconstruction as the number of channels increases. We generate signals with bandwidth $2 \Omega$ by summing cosines of random amplitudes and random frequencies contained within the allowed bandwidth. We reconstruct the signals using $M$ channel TEMs for $M=1,2,6$. We then compute the average of the mean squared error normalized by the signal energy. If this normalized average is below $10^{-3}$, reconstruction is deemed successful, otherwise it is deemed unsuccessful.

\section{CONCLUSION}

We have explored both iterative and closed-form algorithms for the recovery of bandlimited signals encoded using $M$ TEMs, and showed that the inter-integrator shifts do not need to be known. We showed that the reconstructible bandwidth can scale by a factor of $M$ when moving from the single channel case to the $M$-channel case.

In future, we believe that we can show that this scaling factor is actually independent of the shifts between the machines (as long as they are nonzero), and hope to study reconstruction stability when the unknown shifts approach zero. 


\section{REFERENCES}

[1] Claude Elwood Shannon, "Communication in the presence of noise," Proceedings of the IRE, vol. 37, no. 1, pp. 10-21, 1949.

[2] Michael Unser, "Sampling - 50 years after shannon," Proceedings of the IEEE, vol. 88, no. 4, pp. 569-587, 2000.

[3] Yue M Lu and Minh N Do, "A theory for sampling signals from a union of subspaces," IEEE Transactions on Signal Processing, vol. 56, no. 6, pp. 2334-2345, 2008.

[4] Martin Vetterli, Pina Marziliano, and Thierry Blu, "Sampling signals with finite rate of innovation," IEEE Transactions on Signal Processing, vol. 50, no. 6, pp. 1417-1428, 2002.

[5] Hans G Feichtinger and Karlheinz Gröchenig, "Theory and practice of irregular sampling," Wavelets: Mathematics and Applications, vol. 1994, pp. 305-363, 1994.

[6] Raymond Edward Alan Christopher Paley and Norbert Wiener, Fourier transforms in the complex domain, vol. 19, American Mathematical Soc., 1934.

[7] Animesh Kumar, "On bandlimited signal reconstruction from the distribution of unknown sampling locations," IEEE Transactions on Signal Processing, vol. 63, no. 5, pp. 1259-1267, 2015.

[8] Golnoosh Elhami, Michalina Pacholska, Benjamín Béjar Haro, Martin Vetterli, and Adam Scholefield, "Sampling at unknown locations: Uniqueness and reconstruction under constraints," IEEE Transactions on Signal Processing, vol. 66, no. 22, pp. 5862-5874, 2018.

[9] Emmanuel J Candès, Justin Romberg, and Terence Tao, "Robust uncertainty principles: Exact signal reconstruction from highly incomplete frequency information," IEEE Transactions on Information Theory, vol. 52, no. 2, pp. 489-509, 2006.

[10] Anthony N Burkitt, "A review of the integrate-and-fire neuron model: I. homogeneous synaptic input," Biological Cybernetics, vol. 95, no. 1, pp. 1-19, 2006.

[11] Aurel A Lazar and László T Tóth, "Perfect recovery and sensitivity analysis of time encoded bandlimited signals," IEEE Transactions on Circuits and Systems I: Regular Papers, vol. 51, no. 10, pp. 2060-2073, 2004.

[12] Aurel A Lazar, "Multichannel time encoding with integrate-and-fire neurons," Neurocomputing, vol. 65, pp. 401-407, 2005.
[13] Aurel A Lazar, "Population encoding with hodgkinhuxley neurons," IEEE Transactions on Information Theory/Professional Technical Group on Information Theory, vol. 56, no. 2, 2010.

[14] Athanasios Papoulis, "Generalized sampling expansion," IEEE Transactions on Circuits and Systems, vol. 24, no. 11, pp. 652-654, 1977.

[15] Aurel A Lazar and Eftychios A Pnevmatikakis, "Faithful representation of stimuli with a population of integrate-and-fire neurons," Neural Computation, vol. 20, no. 11, pp. 2715-2744, 2008.

[16] Hojjat Akhondi Asl, Pier Luigi Dragotti, and Loic Baboulaz, "Multichannel sampling of signals with finite rate of innovation," IEEE Signal Processing Letters, vol. 17, no. 8, pp. 762-765, 2010.

[17] Aurel A Lazar, "Time encoding with an integrate-andfire neuron with a refractory period," Neurocomputing, vol. 58, pp. 53-58, 2004. 